\title{
Inversão geoelétrica 1D utilizando o algoritmo genético com a determinação do número ótimo de camadas horizontais
}

Gean Lucas Mello Farias ${ }^{1}\left({ }^{\star}\right)$ e Cosme Ferreira da Ponte Neto² / Observatório Nacional - MCTIC

1 -geanfarias@on.br, 2 -cosme@on.br

Copyright 2019, SBGf - Sociedade Brasileira de Geofísica

This paper was prepared for presentation during the $16^{\text {th }}$ International Congress of the Brazilian Geophysical Society held in Rio de Janeiro, Brazil, 19-22 August 2019.

Contents of this paper were reviewed by the Technical Committee of the $16^{\text {th }}$ International Congress of the Brazilian Geophysical Society and do not necessarily represent any position of the SBGf, its officers or members. Electronic reproduction or storage of any part of this paper for commercial purposes without the written consent of the Brazilian Geophysical Society is prohibited.

\section{Abstract}

This work has as main objective to generate a computational routine, a genetic algorithm, to create horizontal geoelectric models that explain the data obtained in different types of electrode arrangements. To do this, the FORTRAN90 programming language will be used, as well as well-known function routines that will aid in the development of modeling. The number of layers of the model will be determined by statistical criteria.

\section{Introdução}

Os princípios físicos do método geoelétrico são conhecidos desde o século XVIII, com a descoberta da resistividade elétrica das rochas, a aplicação do método em prospecção mineral data do início do século $X X$ (ORELLANA, 1972). Atualmente, o método geoelétrico é amplamente utilizado na investigação de geofísica rasa, podendo ser utilizado para delinear sequências sedimentares e descontinuidades verticais que envolvam contraste de resistividade entre as estruturas (TELFORD et al., 1990). Os levantamentos podem ser 1D, do tipo Sondagem Elétrica Vertical - SEV, levantamentos 2D e 3D com "caminhamento" e perfilagem em poços. Existem diversas configurações de eletrodos para os levantamentos: Wenner, Schlumberger, polo-dipolo, dipolo-duplo (KEAREY et al., 2009). A escolha do arranjo depende do problema a ser estudado e dos objetivos do trabalho. O método é particularmente eficiente na localização de aquíferos e, mais recentemente, tem sido muito utilizado em problemas de geofísica ambiental, tais como: caracterização e localização de pluma de contaminação do solo, contaminação de lençóis freáticos, caracterização de agentes contaminantes em subsuperfície e na determinação da cunha salina nas regiões costeiras (ZOHDY et al., 1974) e (GANDOLFO, 2007). Os levantamentos geoelétricos são de logística simples e de baixo custo, as medidas não sofrem grande influência de ruídos eletromagnéticos, permitindo que os trabalhos sejam realizados em zonas urbanas e rurais, os levantamentos não geram grande impacto ambiental e os equipamentos são de baixo custo. Esses motivos contribuíram muito para a longevidade e contínuo aprimoramento do método. Uma limitação é a profundidade pequena de investigação, até $500 \mathrm{~m}$, uma vez que para aumentar a profundidade de investigação é necessário equipamentos de alta potência e uma grande extensão de cabos para os eletrodos. A influência nas medidas de estruturas com contraste de resistividade em profundidades maiores que $1.000 \mathrm{~m}$ é de difícil interpretação.

O projeto proposto tem por objetivo geral resolver o problema de se estimar as resistividades e espessuras verdadeiras de um meio verticalmente estratificado, dadas as resistividades aparentes medidas no meio em estudo. Para tal, a primeira prioridade foi desenvolver um software de modelagem numérica para o método geoelétrico, nos diversos arranjos de eletrodos usuais, para a interpretação de dados em ambientes estratificados. Por último, desenvolver, como ferramenta alternativa para as inversões geofísicas tradicionais usadas na geofísica, um programa que faça a inversão dos dados por aplicação do algoritmo genético.

Os algoritmos genéticos são algoritmos de busca baseados na seleção natural e na genética biológica que exploram de forma eficiente informações históricas para melhorar a eficiência de sua busca pela melhor solução (GOLDBERG, 1989). Esse processo de busca consiste em avaliar em um conjunto de indivíduos, ou seja, uma população de candidatos a soluções, gerados inicialmente por processo aleatório, qual o que melhor se ajusta as condições fornecidas pelo usuário. As melhores características da população anterior são herdados pela geração seguinte, fazendo valer os critérios de seleção em analogia a seleção natural: a nova geração não mais tem componentes totalmente aleatórios, mas tem como base as características dos indivíduos melhores avaliados das gerações anteriores.

Mais detalhes importantes do algoritmo genético, como operadores de mutação e crossover, são discutidos com mais detalhes em GOLDBERG, (1989) e em BOSCHETTI et al., (1996). Detalhes adicionais, como operador de elitismo, que serve para aumentar a velocidade de convergência do algoritmo para soluções com o melhor ajuste, são discutidas em CHAKRABORTY and CHAUDHURI, (2003).

\section{Método}

A formulação do problema geoelétrico começa com o cálculo do potencial elétrico (V) num ponto $\mathrm{P}$ nas imediações de um ponto $\mathrm{O}$, onde é injetada uma corrente elétrica I (figura 1). 


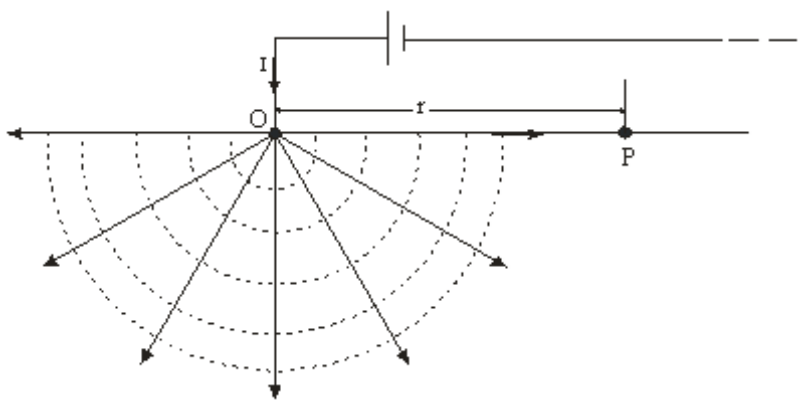

Figura 1 - Fluxo de corrente em subsuperfície de um único eletrodo na superfície.

A solução do problema para $n$ camadas horizontais num semi plano infinito será resolvido através da solução da integral de Stefanescu (figura 2).

$$
\frac{\rho_{1} I}{2 \pi} \int_{0}^{\infty} K(\lambda) J_{0}(\lambda r) d \lambda,
$$

Figure 2 - Integral de Stefanescu.

Onde, $\boldsymbol{\rho}_{1}$ é a resistividade da camada do topo, $\boldsymbol{I}$ é a corrente injetada na superfície, $\boldsymbol{K}$ é a função Kernel, Jo é a função de bessel de ordem 0 .

A função kernel ( $\boldsymbol{K}$ ) pode ser desenvolvida de modo relativamente simples para qualquer número de camadas usando-se relações de recorrência (KOEFOED, 1979) que, progressivamente, adicionam os efeitos de sucessivas camadas na sequência (KEAREY et al., 2009).

Após a formulação do problema geoelétrico para $\mathrm{N}$ camadas horizontais homogêneas, e sua implementação em FORTRAN 90, o algoritmo genético estava pronto para receber os dados observados, sejam eles sintéticos ou reais.

Tradicionalmente, problema inverso geofísico envolve processos dispendiosos computacionalmente, como o cálculo de derivadas e inversão de matrizes. O algoritmo genético dispensa essas operações, além de possibilitar uma busca global, dispensando também outro problema que pode acontecer nos métodos determinísticos, que é limitar sua busca a algum pico local dentro do espaço de soluções. Abaixo encontra-se imagem com um fluxograma simplificado do funcionamento do algoritmo aplicado no presente trabalho.

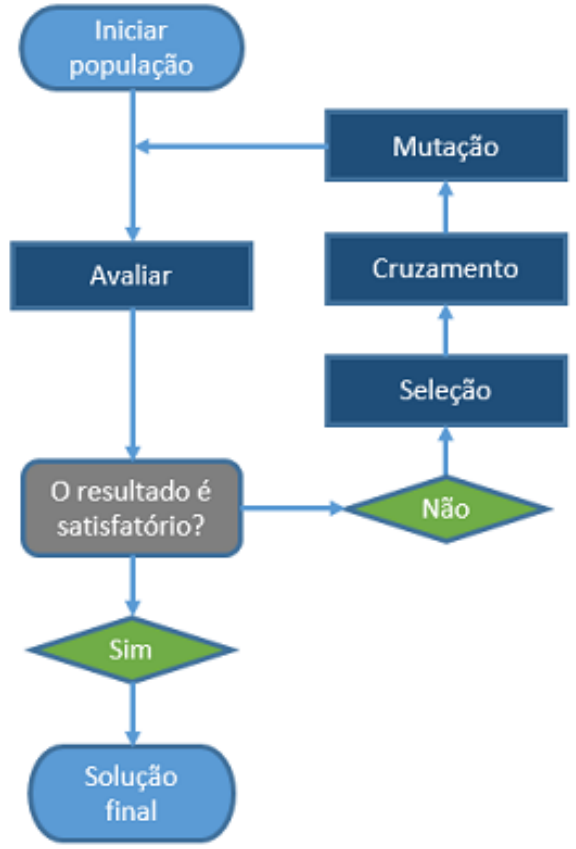

Figure 3 - Fluxograma simplificado do algoritmo aplicado no presente trabalho. Este fluxograma omite o operador elitismo, que foi usado no programa de inversão deste trabalho.

\section{Resultados}

Os dados utilizados neste trabalho foram retirados de CAVALCANTI, (1979). São dados de SEV adquiridos em trabalho de campo realizado na Ilha do Marajó em 1976. O dado escolhido corresponde a SEV 19 do trabalho citado. Segundo o autor, o local é caracterizado por um paleocanal arenoso próximo a superfície, que, por possuir água potável de baixo nível de salinidade, tem alta resistividade. Os valores mais baixos de resistividade na superfície correspondem a areias com cobertura de argila, e os valores mais altos correspondem ao reservatório arenoso contendo água potável. O restante da seção varia entre areias argilosas, areias com água salobra ou conteúdo predominantemente argiloso.

Neste trabalho, foram feitas inversões para diferentes números de parâmetros a fim de, com o teste do quiquadrado $\left(\boldsymbol{X}^{2}\right)$, avaliar a melhor solução encontrada pelo algoritmo genético. No caso, foi testado os modelos de 2 , 3, 4 e 5 camadas horizontais. Os espaços de busca para o algoritmo genético foram pensados para cumprir com as condições dadas pelas informações conhecidas do local.

A escolha da hipótese mais provável vai ser feita através da função distribuição de $\boldsymbol{X}^{2}$, que relaciona a probabilidade da hipótese com o $X^{2}$ e o grau de liberdade do ajuste. Quanto maior o número de camadas do modelo, menor será o grau de liberdade do ajuste e menor será $0 \boldsymbol{X}^{2}$. O equilíbrio entre estas relações inversas é estabelecido pela função distribuição de $\boldsymbol{X}^{2}$. As rotinas para o cálculo da função distribuição de $\boldsymbol{X}^{2}$ estão em PRESS et al. (1996). 
Abaixo encontra-se os resultados dos testes para os diferentes números de camadas, seus graus de liberdade $(\boldsymbol{\eta})$, o $\boldsymbol{X}^{2}$ e a probabilidade $\left(\boldsymbol{P}_{\boldsymbol{\eta}}\right)$ associada a cada modelo ou número de camadas.

Tabela 1 - Tabela mostrando os resultados dos testes de hipótese por $\boldsymbol{X}^{2}$. Note que a probabilidade para o modelo de 4 camadas é a maior; portanto, a mais provável

\begin{tabular}{|c|l|l|c|}
\hline$n^{0}$ de camadas & $\eta$ & $\chi^{2}$ & $P_{\eta}\left(\chi^{2}\right)$ \\
\hline 2 & 25 & 4191.2 & 0.0 \\
\hline 3 & 23 & 9.61 & 99.34 \\
\hline 4 & 21 & 5.02 & 99.99 \\
\hline 5 & 19 & 7.61 & 99.02 \\
\hline
\end{tabular}

O modelo de 4 camadas foi o que se apresentou como a melhor entre as hipóteses pelo teste do $\boldsymbol{X}^{2}$. Abaixo, encontra-se o resultado da inversão.

Tabela $2-\left(^{*}\right)$ Resultados deste trabalho, que foram obtidos por meio de inversão. ( $\left.{ }^{* *}\right)$ Resultados de CAVALCANTI (1979), que foram obtidos por meio de modelagem direta.

\begin{tabular}{|c|l|l|c|l|}
\hline & \multicolumn{3}{|l|}{ Resultados $\left(^{*}\right)$} & $\left({ }^{*}\right)$ \\
\hline Camada & $\rho($ ohm.m $)$ & $\mathrm{H}(\mathrm{m})$ & $\rho($ ohm.m $)$ & $\mathrm{H}(\mathrm{m})$ \\
\hline camada 1 & 29,6 & 1,4 & 40 & 2,1 \\
\hline camada 2 & 193,5 & 18,3 & 180 & 20 \\
\hline camada 3 & 5,8 & 241,4 & 4 & 220 \\
\hline camada 4 & 32,3 & $\infty$ & 22 & $\infty$ \\
\hline
\end{tabular}

A figura 4 mostra os gráficos dos modelos gerados na inversão para diferentes números de camadas, assim como as observações associadas às suas barras de erro, que foram calculadas por propagações dos erros das leituras dos instrumentos de medida associados ao equipamento de campo.

\section{Conclusões}

O resultado da inversão e da determinação do número ótimo de camadas foi satisfatório, os resultados estão coerentes com a interpretação feita por CAVALCANTI (1979), indicando que esta metodologia é eficiente na interpretação de dados geoelétricos para ambientes estratificados.

\section{Agradecimentos}

Ao programa de pós-graduação do observatório nacional. Cosme Ponte, pela orientação. CNPq, pelo financiamento.

\section{References}

BOSCHETTI, F., DENTITH, M.C., LIST, L.D. Inversion of seismic refraction data using genetic algorithms. Geophysics, 61, 1715-1727, 1996.

CAVALCANTI, G.M.L. Geofísica aplicada à prospecção de água subterrânea na área do Rio Paracauari, Ilha do Marajó, Pará. MS Dissertation, Universidade Federal do Pará, Belém, 88 p, 1979.

CHAKRABORTY, B., CHAUDHURI, P. On The use of Genetic Algorithm with Elitism in robust and nonparametric multivariate Analysis. Austrian Journal of statistics, 32, 13-27, 2003.

GANDOLFO, O. C. B. Um estudo do imageamento geoelétrico na investigação rasa. Tese de Doutorado, Universidade de São Paulo, 2007.

GOLDBERG, D. E. Genetic Algorithms in Search, Optimization and Machine Learning (1st ed.). AddisonWesley Longman Publishing Co., Inc., Boston, MA, USA, 1989.

KEAREY, P., BROOKS, M., HILL, I. Geofísica de exploração. Oficina de Textos, 2009.

KOEFOED, O. Geosounding Principles, 1 - Resistivity Prospecting. Gebruder Borntraeger, Berlin, 1979.

ORELLANA, E. "Prospección geoeléctrica en corriente continua". Paraninfo, 1972.

PRESS, W. H., TEUKOLSKY, S. A., VETTERLING, W. T., et al. Numerical Recipes in Fortran 77: The Art of Scientific Computing, v. 1, 1996.

PRESS, W. H., TEUKOLSKY, S. A., VETTERLING, W. T., et al. Numerical Recipes in Fortran 90: The Art of Parallel Scientific Computing. v. 2, 1997.

TELFORD, W. M., TELFORD, W., GELDART, L., et al. Applied geophysics, v. 1. Cambridge university press, 1990.

ZOHDY, A. A., EATON, G. P., MABEY, D. R. Application of surface geophysics to ground-water investigations. Chapter D1, v. USGS Book 2 Collection of Environmental Data, 4th printing, 1974. 


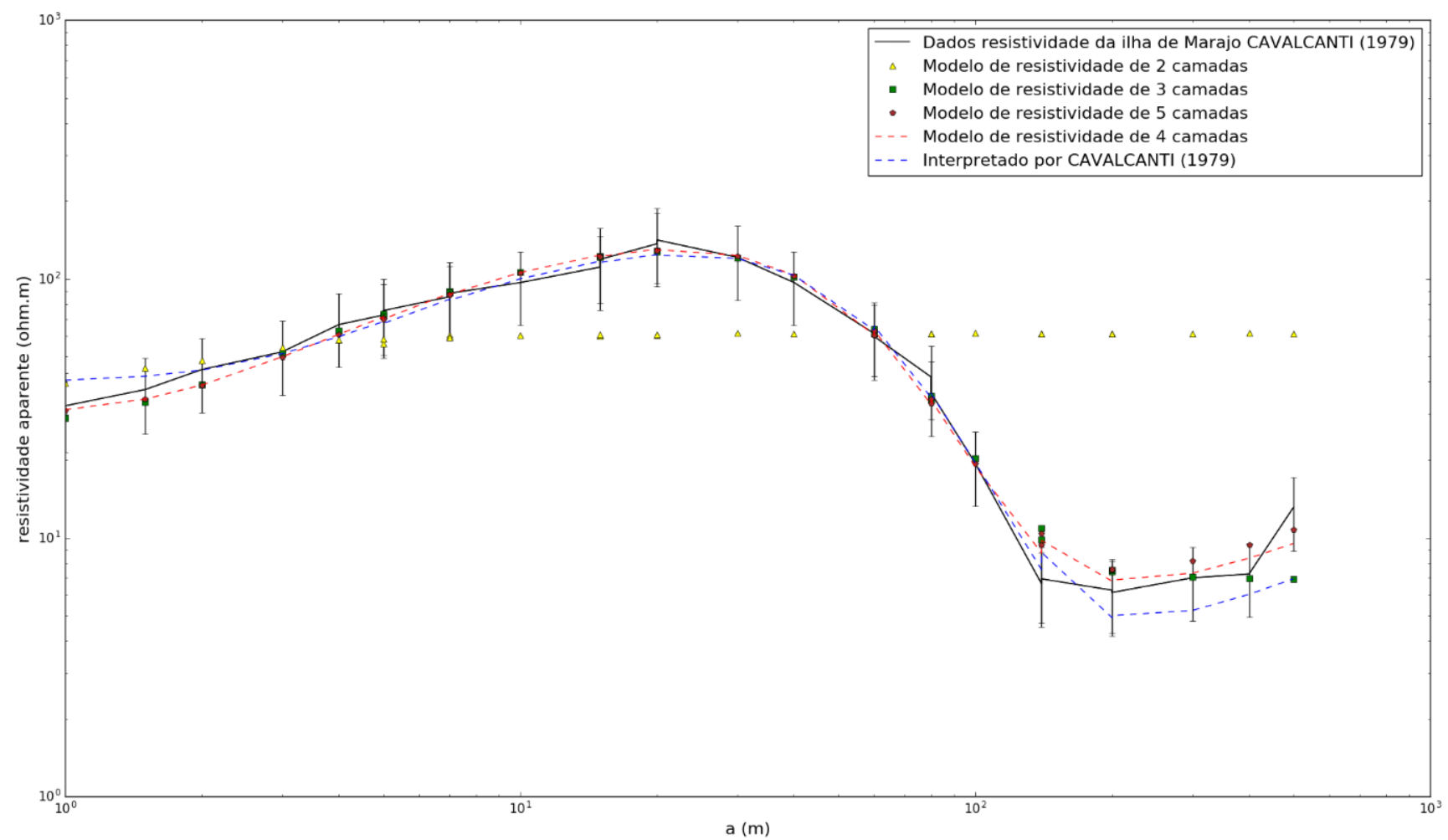

Figure 4 - Gráficos dos resultados deste trabalho, incluindo também o resultado de CAVALCANTI (1979). As barras verticais representam as barras de erro associadas ao dado observado. 